\title{
Graphical Password Authentication by Using Persuasive Click Point Method
}

\author{
Priyanka Gunde ${ }^{1}$, Ujjwala Kokate ${ }^{2}$ \\ Department of Information Technology and Computer Engineering, Sharad Institute of Technology Polytechnic, Yadrav, India
}

\begin{abstract}
Graphical Password essentially uses images or representation of images as password. Human brain is good in remembering picture than textual character. This paper work merges pass points, cued click points and persuasive cued click points. The major goal of this work is to reduce the guessing attacks as well as encouraging users to select more random, and difficult passwords to guess. In this paper, the selection of click points depends upon the method selected by user, selection of sound. Sound gives the security for right click points.
\end{abstract}

Keywords: Text Authentication, graphical passwords, guessing attacks.

\section{Introduction}

Usable security has unique usability challenges because the need for security often means that standard human computer interaction approaches cannot be directly applied. An important usability goal for authentication systems is to support users in selecting better passwords.

The problem associated to vulnerability to shoulder surfing is a major flow that needs to be addressed for current authentication system including graphical password authentication system and traditional alphanumeric password authentication system. According to Webopedia Computer Dictionary, "Shoulder surfing refers to a direct observation, such as looking over a person's shoulder, to obtain information." As define, shoulder surfing usually happen in busy and crowed place, where the person standing behind would try to peek over your back in order to obtain private Motivation of Research Weakness of Textual Password.

So we implement graphical password method. In this paper, the selection of click points of method persuasive click point method, selection of sound. Sound gives the security for right click points.

\section{Introduction}

An important usability goal for authentication systems is to support users in selecting better passwords for security purpose. Semi users often create memorable password that are easy for attackers to guess, but strong system-assigned password are difficult for semi users to remember. Graphical passwords essentially use images or representation of images as password. Human brain is good in remembering picture than textual character. The major goal of this work to reduce the guessing attacks as well as encouraging users to select more random, and difficult password guess. Well known security threats like brute force attacks and dictionary attacks can be successfully abolished using this method. The knowledge based techniques are the most wanted techniques to improve real high security i.e. Recognition based and recalls based.

\section{Recall Based Technic}

There are types of click based graphical password techniques:

1) Pass Points (PP)

2) Cued Click Points(CPP)

3) Persuasive Cued Click Points (PCCP)

The third method is the more secure than other two methods. This method contain two types of security view port with click points on image.

\subsection{Persuasive Click Point}

In Persuasive Cued Click Points method [1],[2],[3],[4],[5] we provide small vies port area which is changed in size that is position on image, therefore user must select click point inside the view port. If user is unable to select click point within a view port then user press shuffle button.

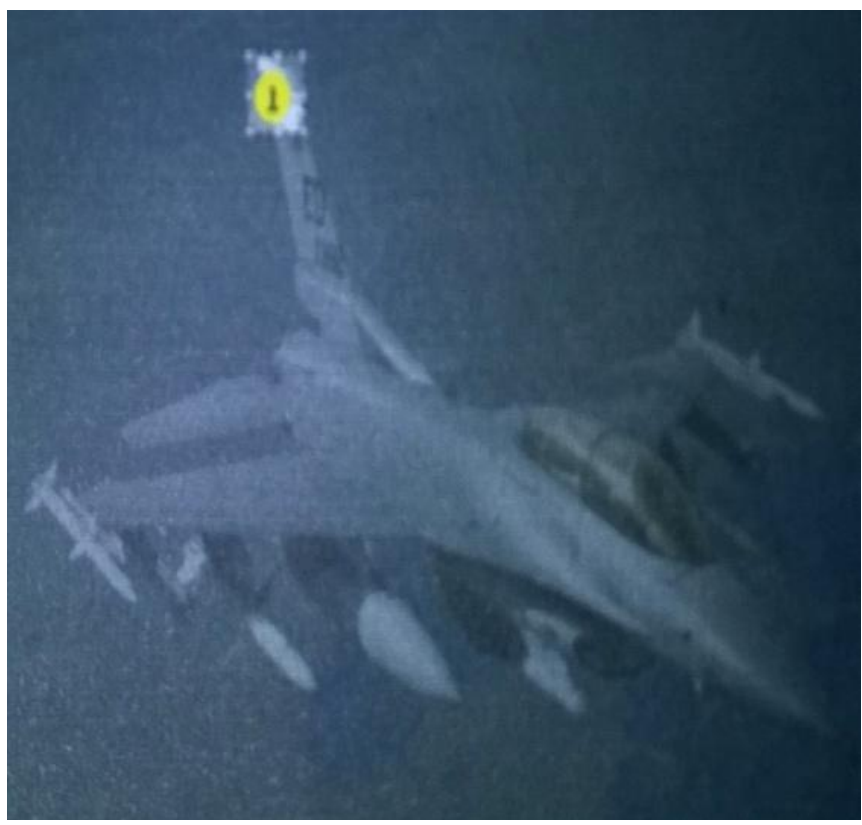

Figure 3.1.1: Persuasive Cued Click Point 


\section{International Journal of Science and Research (IJSR) \\ ISSN (Online): 2319-7064}

Index Copernicus Value (2013): 6.14 | Impact Factor (2014): 5.611

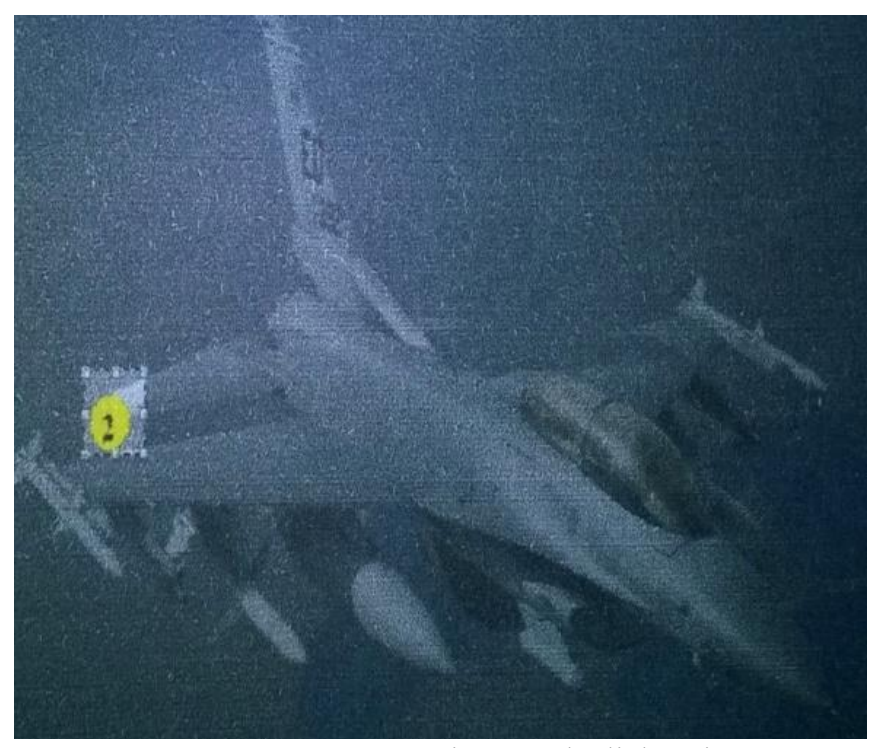

Figure 3.1.2: Persuasive Cued Click Point

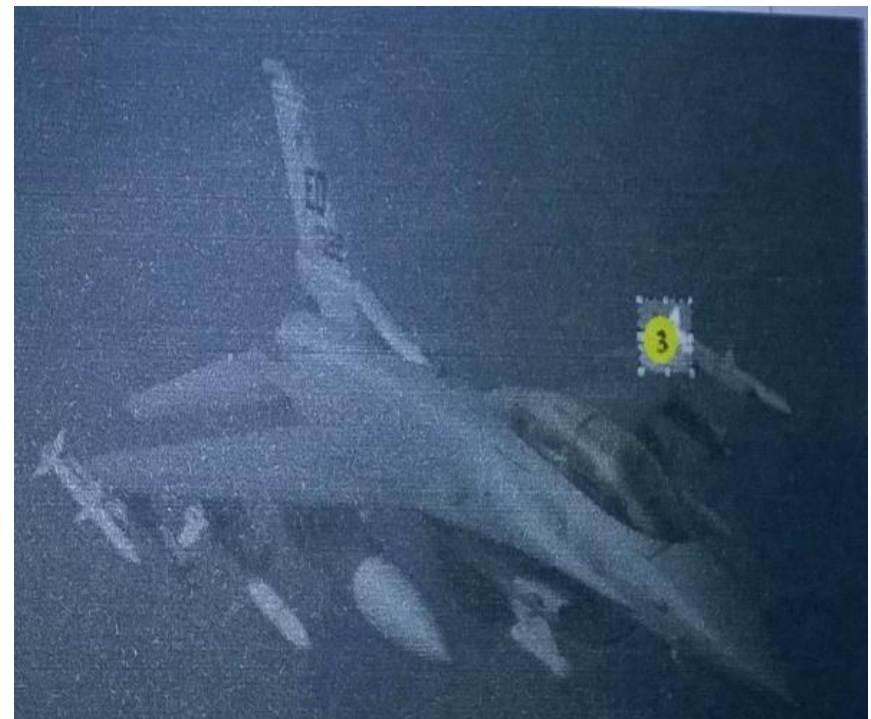

Figure 3.1.3: Persuasive Cued Click Point

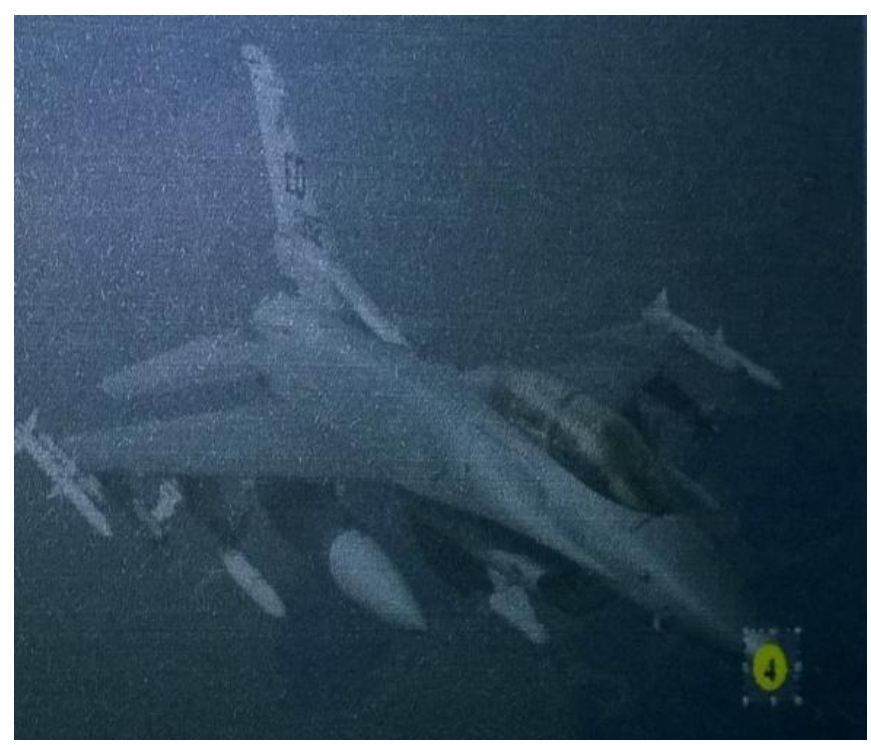

Figure 3.1.4: Persuasive Cued Click Point

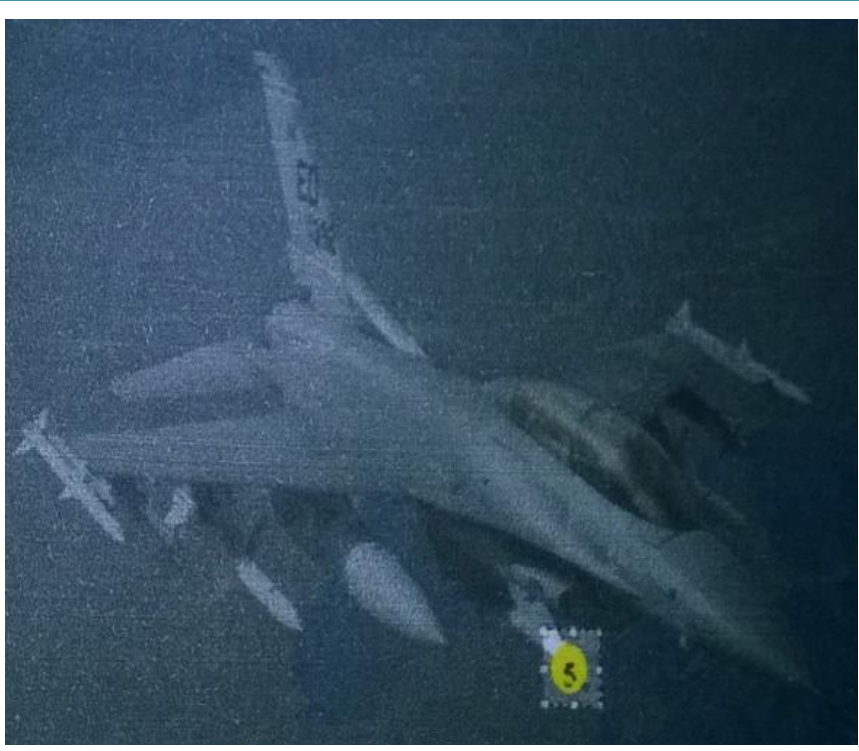

Figure 3.1.5: Persuasive Cued Click Point

\subsection{Sound Selection}

For the registered user identification purpose in this paper we include one right click point play correct sound which is selected at the time of registration. Security is checked on view port size, click points and correct sound selected at the time of registration.

\section{Data Analysis}

\subsection{Graphical Analysis}

Graphical analysis simply means displaying the data in a variety of visual formats that make it easy to see patterns and identify differences among the result set. To recognize the click points $\mathrm{X}$-axis $\mathrm{Y}$-axis are used. At the time of registration user select the click point on image is recognize by ImageClickeventArgs class. This class provide data for any event that occur when a user clicks on image based asp.net server control, such as HtmlInputImage or ImageButton server controls. While clicking an ImageButton server control causes a click event occur. At the time of login when user click on image to recognize click Math class is used. Math Class provides constant and static methods for Trigonometric, Logarithmic and other common mathematical function. Math.Abs() method returns the absolute value of a specified number.

\section{Conclusion}

By using Persuasive cued click point scheme is its large password space over alphanumeric passwords. There is a growing interest for Graphical passwords since they are better than Text based passwords, although the main argument for graphical passwords is that people are better at memorizing graphical passwords than text based passwords. Online password guessing attacks on password only systems have been observed for decades. "Present day attackers targeting such systems are empowered by having control of thousand to million node botnets. 


\section{References}

[1] [IJESAT] Defenses Against Large Scale Online Password Guessing Attacks By Using Persuasive Click Points Abdul Rasheed. Sk 1, Madhava Naidu.V 2, D.sunitha 3

[2] Persuasive Click Points Based Large Scale Online Password Guessing Attacks K. Hari Krishna

[3] International Journal Of Computer Engineering\& Technology (Ijcet) "Defenses Against Large Scale Online Password Guessingattacks By Using Persuasive Click Points" Mrs. M. A. Patel1, Ms. Y.U.Kadam2, Ms. R. Y.Thombare3, Ms. H. P. Patil4

[4] International Journal of Computer Science and Mobile Computing "Defenses against Large Scale Online Password Guessing by Using Persuasive Cued Click Points"

[5] IOSR Journal of Computer Engineering (IOSR-JCE) "Online Password Guessing Attacks by Using Persuasive Click Point with Dynamic User Block” P. Kalaivizhi11, Dr. S. Thiru Nirai Senthil2 